\title{
GIS AND BIM INTEGRATION AT DATA LEVEL: A REVIEW
}

\author{
Mohammed Jawaluddeen Sani ${ }^{1,2^{*}}$ and Alias Abdul Rahman ${ }^{1,}$ \\ ${ }^{1}$ 3D GIS Research Lab., Faculty of Built Environment and Surveying, Universiti Teknologi Malaysia (UTM), 81310 UTM \\ Skudai, Johor, Malaysia \\ alias@utm.my \\ ${ }^{2}$ Department of Surveying and Geoinformatics, Federal Polytechnic, Bauchi, Bauchi State, Nigeria \\ * mjsani71@gmail.com
}

KEYWORD: GIS, BIM, CityGML, IFC, and data Integration models.

\begin{abstract}
:
City Geography Markup Language (CityGML) and Industry Foundation Class (IFC) are the two most popular data exchange format for the integration of Geographic Information System (GIS) and Building Information Modelling (BIM) respectively and has been identified by many researchers as an auspicious means of data interoperability between the two domains but with challenges on the compatibility between them. The main issue is the data loss in the process of information transformation. The success of integrating these two domains (GIS and BIM objects) is a great achievement toward solving problems in Architecture, Engineering and Construction (AEC), Facility Management (FM), Disaster Management (DM) sectors. Nevertheless, as we all know GIS and BIM are different fields used by different professionals using different software packages, used for different purposes, it is definitely face with many challenges including data interoperability, mismatch and loss of semantic information are bound to occur during the process of integration. In order to comprehend the two domains and their data models of CityGML and IFC. This paper review existing models on GIS and BIM developed by different researchers, the complementarity and compatibility of GIS and BIM on the previous integration techniques were also reviewed and finally, the paper review the integration of GIS and BIM at the data level aimed at solving different problems surrounding it by considering the transformation of coordinates at geometric level from CityGML to IFC, in order to achieve flow of information between GIS and BIM.
\end{abstract}

\section{INTRODUCTION}

Geographic information system (GIS) and Building Information Modelling (BIM) are two different domains use for the interpretation of 3D model from different perspectives, virtually using two different most popular data exchange formats. For instance, City Mark-up language (CityGML) and Industrial foundation class (IFC) respectively. GIS concentrate on the geographical information of buildings and its components from geographical viewpoints. On the other hand, BIM focuses more on the detailed building components and project information, like cost and schedule from an architectural and construction viewpoints (Liu et al., 2017). It is a field widely accepted by Architecture, Engineering and Construction (AEC) as a result of advancement in technology which has increasingly enriched the construction industry over the years, as it allowed stakeholders to capture, manipulate, update and exchange information throughout the building construction project lifecycle (Fosu et al., 2015). BIM serves as an intermediary of interoperability among industries' players thereby boosting its popularity among clients (Azhar et al., 2015), GIS on the other hand visualizes and analyses location related issues in geospatial science and natural resource management by integrating different spatial and attribute data and deriving knowledge through diverse spatial analysis tools and modelling modes (Zhu et al., 2018). This pave way for spatial features or data to be stored as geometric and referenced with coordinates and map projections. GIS has over the years been used with $2 \mathrm{D}$ mapping in order to analyse data over large areas (Zhu et al., 2018); F. Biljecki et al., 2016). Moreover, as a result of advancements in technology the principles of the 2D GIS are now applied to 3D spatial data for more complex documentation, visualization and analyses (Dore \& Murphy, 2012). As 3D GIS is fast becoming bases for decision making for many applications. Examples of such applications are: site selection for (solid and liquid waste management, solar installations), urban and regional planning, environmental and health simulations, crisis and disaster management. The integration of GIS and BIM is the merging of the two systems for the purpose of visualisation and analysis.

Recently, a series of reviews on GIS-BIM integration were presented (Zhu et al., 2018; Liu et al., 2017; Song et al., 2017). For instance Zhu et al (2018) conducted a critical review on GIS and BIM integration at the data level while focused more on the following: 1. Identifying most relevant models employed for the integration and highlighted their strength and limitations; 2 . Considered the potentialities of other models; 3. Provided future directions on GIS and BIM data integration: Similarly, Liu et al (2017), reviewed the state-of-the-art on GIS and BIM integration by concentrating more on the various reason for which the integration methods were meant for as well as the parameters that influenced the selection of the methods according Effort, Flexibility, Extensibility and Effectiveness (EEEF). Likewise, Song et al (2017) reviewed the integration of GIS and BIM from spatio temporal statistical perspective in the AEC industry; which focused mainly on the GIS and BIM integration methods skills, mathematical modelling and data analysis. In order to compliment these efforts, this review will focus on integration at data interoperability levels while focusing on integration of GIS and BIM using data exchange formats/schemas of the two domains, CityGML and IFC for GIS and BIM respectively.

However, in order to present the clear picture of the integration, research was conducted and came up with a clear understanding of their key components. This study examined the possibility of 
developing a model that can be used to integrate the two systems. Furthermore, papers that carried out the review on GIS or BIM independently (without the integration as a link between the two domains) were not considered in the selection since this review focuses more on GIS and BIM integration, particularly the data models of GIS and BIM. Moreover, it is acknowledged that the GIS and BIM integration base on methods or models reviewed here are widely selected, even though new ones are developing on regular bases. Hence, this review will only offer the broad category of both the models on GIS and BIM integration that are readily available online at the time of this study.

This review is structured as follows. Section 2 description of some previous works on GIS-BIM integration. Section 3 . Integration of GIS and BIM at the data levels (using data schemas of the two domains, IFC and CityGML for BIM and GIS respectively). Section 4. Discussion, and section 5. Conclusion and future works.

\section{DESCRIPTION OF SOME PREVIOUS WORKS ON GIS - BIM INTEGRATION}

Previous researches on BIM and GIS integration concentrated more on the integration technologies, in order to come up with the best and efficient means of the integration. To address the problems of GIS-BIM integration various approaches and models were proposed (Song et al., 2017; Liu et al., 2017; Peuwela et al., 2017; Niu et al., 2015; Amirebrahimi et al., 2016a). For the integration pattern of GIS-BIM, many researchers preferred unidirectional approach (extracting data from BIM to GIS, and others from GIS data to BIM), some researchers' proposed new tools, extensions, ontology and framework while others concentrate on bidirectional approach (Unified Building Model (UBM)). For instance, CityGML and IFC are the most popular and clear data exchange formats for exchanging geometry and semantic data for GIS and BIM, mainly referred to as the primary standards for GIS and BIM integration (Gröger \& Plümer, 2012; Song et al., 2017) . In the process of integration, there is information loss as a result of the extraction process and simplification of data from one system to another (Wu \& Zhang, 2016). In order to avoid information losses, El- Mekawy et al (2012) presented UBM.

\subsection{Complementarity of GIS and BIM}

The two domains were developed for different purpose and to work on different platform but yet complement each other through data exchange. In essence, GIS has been developed to manipulate, manage, visualize and analyze spatial data, based on geometric technologies. As the system allows the storage of spatial information in the relational - object oriented database, GIS is not limited to only data storage system it goes beyond that. The attribute data related or linked with spatial features are stored in the database which allow for spatial analysis (Liu et al, 2017).

BIM is relatively rich and detailed in construction information for both geometry and semantic information, however, it does not include the surrounding information (Xiong et al., 2013; Steed et al., 2012). The limitations of BIM in the spatial planning for construction is clearly identified by ( $\mathrm{Li}$ et al., 2016; Yang et al., 2017). Most of the time, spatial information which is necessarily needed by BIM system for resource arrangement, safety analysis, environmental evaluation, and environmental impact analysis (Kang and Hong, 2013). For instance, topographic information of an area, which is vital to the spatial planning of gravity dam construction, can only be found in GIS. Optimization of tower cranes' location on construction site is another classic example that BIM requires spatial information (Irizarry and Karang, 2012; Irizarry et al., 2013). The good relationship that exist between GIS and BIM is clearly depicted in the Figure 1 even though, the two technologies have matured in a diverse ways. With the recent high demand for merging indoor and outdoor applications for diverse purposes, efforts have being made to design systems and tools to integrate building models within a geospatial framework (Liu et al., 2017). The complementarity and diverse nature of GIS and BIM emphasises their integration capabilities.

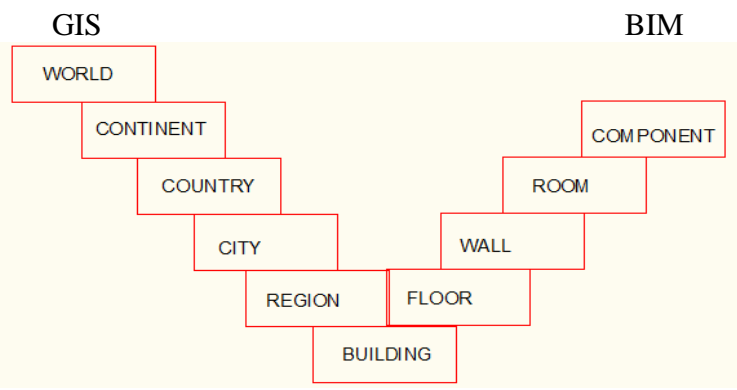

Figure. 1. Overlap between GIS and BIM

The integration of GIS and BIM warrant deep insight for better decision making, communication and understanding. Using BIM with GIS couple with time information 4 dimension (4D), built environment can better understand the essence of decisions before, during and after the construction project. GIS and BIM integration system allows an effective management of information in various stages of a projects' life cycle, ranging from planning, design, construction, operation, and maintenance. Information at any spatio-temporal scale can be available for different applications in such system. Effective management of various information provide vital supports for decision-making from different sources (Xie et al., 2017). The integration of GIS and BIM is a vital tool in the development of smart sustainable city due to its abilities, applications of technologies, data integration, urban management and quantitative analysis (Ma \& Ren, 2017). As smart sustainable city is regarded as widely used technology for the enhancement of urban life sustainability, which requires enormous and multisource data for management and technologies use (Song et al., 2017).

The integration of GIS and BIM created strong synergy due to the complementarity of one another. GIS is an expansive field of study that covered geospatial modelling and geovisualisationbased decision making (Gale et al., 2016), while BIM has advantages on rich geometry and semantic information through the life cycle of the building (Volk et al., 2014). The advantages of GIS and BIM were discussed in some of the previous literatures respectively (Ma \& Ren, 2017; Liu et al., 2017; Pauwels et al., 2011). The essence of integrating GIS and BIM is to integrate the robust parts of both domains for building and $3 \mathrm{D}$ city modelling. Few years back, the integration has been applied on multiple cases; for instance, visualization of construction supply chain management, emergency response, urban energy assessment and management, heritage protection, climate adaption and ecological assessment, just to mention but a few. 
Gone by the days when users tried to find solutions to their problems regarding GIS and BIM integration within their domains (Laat \& Berlo, 2011; Biljecki et al., 2015) where, BIM users extract spatial information in BIM system while GIS users tried to integrate BIM data into a GIS system (unidirectional approach) which lead to semantic information loses (ElMekawy et al., 2012b). Currently the solutions to the problems of GIS and BIM integration is efficient and successful data interoperability.

Previous researches had clearly depicted the potentiality of GIS data on facilities management, by identifying the information that are more relevant as both GIS and BIM uses spatial information were BIM provides indoor 3D model geometry and semantic information while GIS provides outdoor modelling information. The key function that make this work, is exchange and use of information (data interoperability) between GIS and BIM by using the platform appropriately. Effective facilities management requires data collection spatio-temporally. This cannot be achieved by relying entirely on 3D information which is gathered from the model. Additional inputs from different data with a BIM object, is required to manage and process data. Effective integration of GIS into any facility which used BIM for facility management (FM) can provide the operator with facility management data for effective management of FM processes (Kang \& Hong, 2015).

GIS and BIM integration is a topic that need to give more attention because systems need to integrate with one another for a better decision making. As said by Heikikila published way back in 1998 where he stated that "The 'core' of the computing universe will continue to shift dramatically from the desktop to stand-alone CPU to the network, and planners will not be exempt from this pervasive trend." (Hingmire \& Thomas, 2017).

\subsection{Level of integration}

Different levels of integration for GIS-BIM integration were proposed by (Karang and Hong, 2015; Irizarry et al., 2013; Amirebrahimi et al., 2016). Where, Karang and Hong, (2013) grouped the levels into five based approaches as; processes, ontology, schema others are, system and service based approaches. Irizarry et al, (2013) grouped them into two levels, namely: application and fundamental levels. The former focus more on the creation or development of the methods that harness the potentials of the two systems (GIS and BIM) for the integration and the later concentrate more on the data interoperability and data exchange at the data level. Moreover, Amirebrahimi et al, (2016) categorised them into three groups namely: data, application, and process levels. At the data level the data models and structure are manipulated to meet the requirements of the application in question and to some extent, they are extended to achieve the goal of the application. Application level adopts new applications that harness functionalities of GIS and BIM. This level could only be built on a successful and efficient data interoperability. At the process level both the two systems (GIS and BIM) are employed in a workflow and integrate. However, the most difficult to achieve is the application level and its success solely rely on the success of data interoperability. Furthermore, the most prominent and difficult to achieve among them as categorised by Amirebrahimi et al., 2016: is the integration at the data level, therefore proper attention should be given. However, this research should concentrate more on the integration at the data level.

\section{INTEGRATION OF GIS AND BIM AT THE DATA LEVELS.}

\subsection{Standard data models}

Integration of GIS and BIM at the data level, simply means data interoperability between GIS and BIM. The data structure or formats should always be taken into consideration, although, there are different data formats in existence that could be used to store 3D geometry but the two most popular among them are CityGML and IFC for GIS and BIM respectively. The GIS and BIM data schemas have different concepts, development purposes, and structures, therefore, making it difficult to integrate data if certain criteria were not given. In this case we tend to analyzes the data schema structure to be considered from the BIM-GIS integration perspectives. There exist commercial data formats in respect of GIS and BIM that is not open. Based on CityGML and IFC, which are the representative standard models of GIS and BIM, the data schema structure and features are analyzed in this section.

3.1.1 City Markup Language (CityGML) :According to Deng et al., (2016), CityGML is an open standard data model and exchange format that has the capability to store 3D models of cities and landscapes based on Geography Markup Language (GML) base, as identified by the Open Geospatial Consortium (OGC) in Extensible Markup Language (XML) format. CityGML is an application schema for GML 3.1.1 (GML3) which is a standard for interoperability between $2 \mathrm{D}$ and 3D geospatial information over the internet (Mignard \& Nicolle, 2014). It is XML-based data models, it defines the basic attributes, relations and entities of a city, which is essential for cost-effective sustainable 3D city model maintenance. CityGML is divided into two parts (1) the schema that describes the document and (2) the instance document that contains the actual data. It has definitions for different Levels of Detail (LODs) in order to reflect the amount of detail included in a model. Building models of a single residential building in LOD1 to LOD4 is presented in Figure 2. Higher LODs determines the contents included in the model, LOD0 shown in the figure as the footprint of the building in 2D: LOD1 models are referred to as the basic block model with flat roofs: LOD2 is also a basic block model with different roof style. As LOD3 and LOD4 models incorporate windows and doors which have close exterior views, while their internal components are quite different. LOD4 contains interior spaces (rooms) and internal walls, while the model in LOD3 does not. However, the building model in CityGML is less complete and mature as in BIM, even in LOD4 (Amirebrahimi et al., 2016).

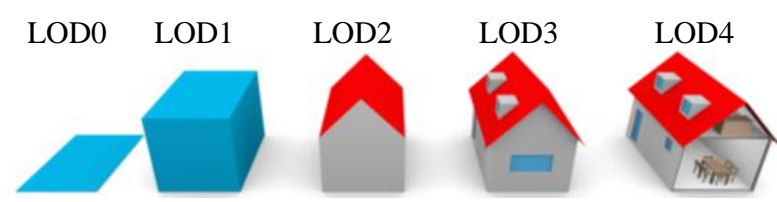

Figure. 2. A building model in CityGML LOD0 - LOD4 (Biljecki et al., 2014a).

3.1.2 Industry Foundation Class (IFC) standard model : The Architecture, Engineering and Construction (AEC) industry is divers therefore information demanding (Aziz et al., 2006), and there are various $3 \mathrm{D}$ data formats in existence from divers vendors that deter information exchange in this field (Atazadeh et al., 2017). Although there are many open BIM standards, such as BIMXML and COINS in existence, IFC is the primary 
open data schema used for information exchange within AEC/FM domains (Amirebrahimi et al,2016), and it is EXPRESS-based which is developed by buildingSMART (formerly: International Alliance for Interoperability (IAI)) in 1994 (Mignard et al,2016).

IFC describes the information schema in the EXPRESS language based on the object-oriented (OO) concept. Focusing on the interoperation of architectural information to enable the reuse of the information, it provides a total of over 700 objects, including various architectural elements, materials, and processes, which are mostly extended from the kernel objects.

IFC classifies BIM models into four groups according to the details they contain by Levels of Development (LODs), from LOD100 to LOD400. Figure 4 shows a building model from LOD100 to LOD400. With the increase of LOD, more details are contained in the model. On LOD100, there is only one solid building in the model, but on LOD400, it has already been a complex model with several components, including interior furniture, interior wall etc. LOD0 example is not given, because LOD0 elements are not geometric representations. Note that LOD 100, 200, 300, and400 are defined by American Institute of Architects (AIA), while LOD 350 is developed by the BIMForum working group (BuildingSmart), as it was found to be necessary to define a LOD between LOD300 and LOD400 for detailed coordination between disciplines, e.g., clash detection/avoidance, layout, etc.

Subsequently, CityGML and IFC are frequently chosen as the representatives data exchange formats for both GIS and BIM respectively (Deng et al., 2016), therefore our discussions regarding data level of GIS-BIM integration will focus on the two systems (GIS and BIM), therefore, the transformation of BIM to GIS will refer to the transformation of IFC to CityGML.

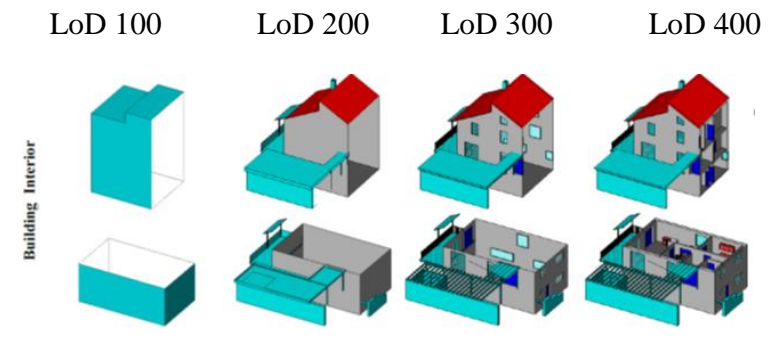

Figure. 3. A building model in IFC from LOD100 to LOD400 (Gröger \& Plümer, 2012).

\subsubsection{Geometry and Semantic Information for both IFC}

and CityGML : Geometry and semantic levels are the two sub-levels of data level of GIS-BIM integration, geometry level focus more on the transformation of information related to geometry while semantic level concentrate on full attribute information transfer.

At the geometry level there are three challenging issues with regard to this, these include: the use of different reference system by the two systems (GIS and BIM), 3D geometry and harmonization of LODs.

GIS as a system that adopt geographic coordinate system (GCS) to cover location (state, region, nation and the world at large) where each object within any of the location has its geographical coordinate (GC) inform of longitude, latitude and height or universal transverse mercator (UTM) inform of northing, easting and height. On the other hand, BIM uses a local placement system where objects are defined using local planar coordinate system, even though GIS uses local planar coordinate system, but in rear cases, the most use by GIS is GCS. The problem of reference system could be solved by the proposed equation (Wu \& Hsieh, 2007) below: where, the vectors are the coordinates of the same object but in different coordinate system (LCS and GCS) the vectors could be transform from one another using coordinate system transformation matrix.

Matrix equation for computing the coordinates of vertices of the objects in the local coordinate system:

$\left[\begin{array}{l}x^{\prime} \\ y^{\prime} \\ z^{\prime}\end{array}\right]=D \cdot\left[\begin{array}{l}V x \\ V y \\ V z\end{array}\right]+\left[\begin{array}{l}x \\ y \\ z\end{array}\right]$

Where D:- is the sweeping distance and $(\mathrm{Vx}, \mathrm{Vy}, \mathrm{Vz})$ :- is the direction vector of sweeping ( $\mathrm{Wu} \&$ Hsieh, 2007).

Matrix equation for transforming coordinates from the local system to the real world system

$$
\begin{aligned}
{\left[\begin{array}{l}
x 2 \\
y 2 \\
z 2
\end{array}\right]=} & {\left[\begin{array}{ccc}
1 & 0 & 0 \\
0 & \cos \theta_{x} & \sin \theta_{x} \\
0 & -\sin \theta_{x} & \cos \theta_{x}
\end{array}\right]\left[\begin{array}{ccc}
\cos \theta_{y} & 0 & -\sin \theta_{y} \\
0 & 1 & 0 \\
\sin \theta_{y} & 0 & \cos \theta_{y}
\end{array}\right] } \\
& {\left[\begin{array}{ccc}
\cos \theta_{z} & \sin \theta_{z} & 0 \\
-\sin \theta_{z} & \cos \theta_{z} & 0 \\
0 & 0 & 1
\end{array}\right]\left[\begin{array}{l}
x 1 \\
y 1 \\
z 1
\end{array}\right]+\left[\begin{array}{l}
\Delta x \\
\Delta y \\
\Delta z
\end{array}\right] }
\end{aligned}
$$

Where: $\theta \mathrm{x}, \theta \mathrm{y}, \theta \mathrm{z}$ : - are the angles of rotations about the $\mathrm{X}^{\prime}$ axis, $Y^{\prime}$-axis, and $Z^{\prime}$-axis, respectively, for making the axes of the $\mathrm{X}, \mathrm{Y}, \mathrm{Z}$ coordinate system parallel to the corresponding axes of the $X^{\prime}, Y^{\prime}, Z^{\prime}$ coordinate system

$\Delta(\mathrm{x}, \Delta \mathrm{y}, \Delta \mathrm{z}):-$ is the translation vector from the origin of the local coordinate system to that of the real world coordinate system. (Wu \& Hsieh, 2007)

For the representation of objects, there are three approaches to 3D geometry representation of object, these include; i. boundary representation (b-rep); 2. Sweep Solid (SS); and 3. Constrictive Solid Geometry (CSG). GIS adopt the use of only b-rep, like CityGML and multipatch. The issue of transformation from $b-$ rep to b-rep, from SS to b-rep and from CSG to b-rep; could be solve by coordinate system transformation function, by customize function and by open computational geometry library respectively. But there are issues still with transformation from b-rep to another system which is now the challenging issue in 3D geometry representation of object (Deng et al., 2016). While BIM, use IFC file that uses one of b-rep, SS and or CGS or takes their combination to represent a 3D geometry.

LODs in both GIS and BIM depict the amount of details in city model and building model respectively. The two systems (GIS and BIM) have the same number of levels, but have diverse definitions for corresponding LODs. For instance, CityGML defines a building model LOD0 as footprint of the building, while an element defines as LOD100 in IFC may not even be a geometry representation (Zhu et al., 2018). 

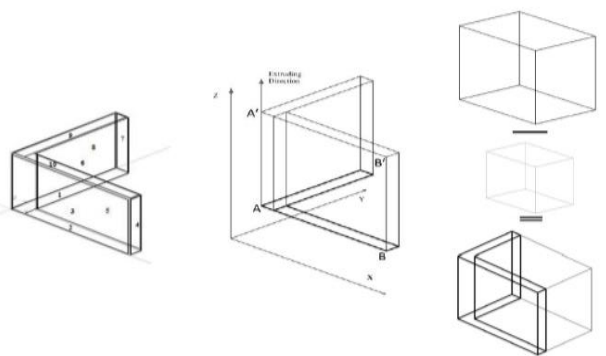

i. B-Representation ii. Swept Solid iii. CSG

Figure. 4. B-Rep, Swept Solid and CSG (Deng et al., 2016)

This is the reason why the LODs cannot easily be mapped. As a result of that a barrier is created for full and successful data interoperability between the two systems (GIS and BIM). In order to achieve successful data interoperability between GIS and BIM several researches were conducted, for instance, Deng et al successfully transformed IFC building to CityGML LOD1 to LOD4 models (Deng et al., 2016). Also, Donkers et al. developed a method to automatically generate CityGML LOD3 building models from IFC file for the construction of a city model (Donkers et al., 2016) however those research were only able to achieved the transformation from IFC to CityGML. Transformation or data exchange from CityGML to IFC is equally important for a successful data interoperability.

Even though, it is too complex, because CityGML has less definition for classes compered to IFC. For instance, there are at least 5 to 6 classes, such as beam, column, and stair that could be mapped with "BuildingInstallation" in CityGML. The mapping of "BuildingInstallation" to the corresponding class in IFC could be difficult, as one will be left with the decision to which class of IFC the "BuildingInstallation" is to be map. Which means, the mapping process may have to be manual prior to the development of a model that will have the ability to categorise, for example, stairs, columns, or beams. However, the main goal of conducting this research is to achieve transformation from IFC to CityGML despite all the challenges.

Geometry transformation between the two systems, could hardly be achieve without employing some commercial software packages , for instance, Feature Manipulation Engine (FME), BIMServer, and Data Interoperability (DI) which is an extension for ArcGIS built on FME. Never the less, none of these tools will be able to transfer both geometry and semantic data between GIS and BIM successfully (Donkers et al., 2016)

The purpose of the integration at this level is mainly for 3D visualization, as usual the main issue is the semantic lose, which is attributed to semantic mismatch between GIS and BIM. For instance, a stair could not be displayed correctly after transformation from IFC to CityGML, as there is no definition for stair in CityGML (Laat \& Berlo, 2011).

There exist the issue of mismatch of semantic information between GIS and BIM, were the two domains have different definitions for the same object, for instance, a door in IFC is defined as "Ifcdoor" while it is just "door" in CityGML; and in some example one of the schemas defines a component while the other one failed to, for instance, IFC defines beam, column, stair, and so on, while CityGML only generalized the component like "BuildingInstallation" (Donkers et al., 2016). Most of the semantic information loss is on the side of GIS, because CityGML contain less information than IFC. The main effort in sematic level data interoperability is to harmonise the two schemas, which call for the modification to the existing schemas (CityGML and IFC). However, different approaches were employed, for instance, simplification, schema extension, or creation of new intermediate schema(s).

The need for simplification approach for data interoperability, is to simplify the complex IFC schema for few specific task, for instance indoor navigation. (Isikdag et al.,2013) developed a BIM Oriented Indoor Model, for the purpose of facilitating indoor navigation. It eliminate solid elements in the building model, such as holes in the slabs and walls, and only keeps the necessary attributes.

For the extension of schemas, this could be sub-divided into; extension from the side of CityGML and IFC extension. The CityGML extension is through Application Domain Extension (ADE), which is a CityGML standard that support ADEs to employ new definitions for new objects. Laat \& Berlo, (2011) defined "stairs" in CityGML, which was not originally defined. Also, IFC could be extended where necessary, as Borrmann et al, (2015) in their research on IFC model for incorporating multi-scale representation of shield tunnels, which was later transformed to CityGML. Thus, this approach may likely across some issues for example visualization. Likewise, CityGMLADE geometry may not be represented correctly in some 3D viewers like Autodesk LandExplorer (Laat \& Berlo, 2011)

The development of an intermediate model or new model will harmonize the current data exchange models (CityGML and IFC). In relation to developing an intermediate data model, all the information of one model will pass through an intermediate schema to the other model. This approach solely relies on semantic web technology, which is the centre of the semantic web ontology, it is a set of technologies for representing, and browsing structural data on web (Hor et al., 2016). However, the development of a new data model, the geometry and semantic from IFC were extracted using DI and then merged using a unique identifier ID of each element, later import into the designed data model. For instance, Urban Flood Model (UFM), which is an XML-based and designed to facilitate micro-level flood assessment (Amirebrahimi, 2015)

Moreover, a unified building model developed base on ontology method, for instance, Karan and Irizarry used this method in an attempt to extend BIM's scope to the preconstruction planning phase by enabling site layout design that tends to be done by GIS (Karan \& Irizarry, 2012). Deng et al., (2016), adopted a similar method for creating a reference ontology called Semantic City Model, which serves as an intermediate model for exchanging information between IFC and CityGML, and with which they achieved mapping between BIM and 3D GIS at different LODs. Costa et al., (2016), developed a District Data Model (DDM), which contains information from IFC and CityGML data as well as contextual data, to support the retrofitting design of energy-efficient districts. Other examples include the UBM, which is designed and tested by (El-Mekawy et al., 2012) using BIMServer that supports bidirectional information exchange between IFC and CityGML in LOD1LOD4, and the Integrated Geospatial Information Model (IGIM) by (Hor et al., 2016). However, the semantic web-based method has its own advantage as it's solve many problems, but time consuming, though it is still developing. 


\section{DISCUSSION}

\subsection{Difference between GIS and BIM integration levels}

As stated earlier, this paper concentrated more on integration of GIS and BIM at the data levels. Different researches classified integration into different classes for levels of integration; like the work of (Karang and Hong, 2015; Irizarry et al., 2013; Amirebrahimi et al., 2016). Amirebrahimi et al, (2016) categorised the integration into three groups namely: data level, application level, and process level. In this study we will consider only data level, application level and process level could be a sub-group under the data level, based on the fact that, the boundary between application and data levels is not diverse. Therefore, it is hard to establish whether a research is at the data level or application level, because research at application level rely on the success of data interoperability. For instance, a comprehensive account on how BIM and GIS data were transformed from IFC or CityGML through Web Ontology Language (OWL) to the formats that may well be used by simulation applications, e g Energy Plus and CitySIM for city simulation (Costa et al., 2016). On the other hand, in a situation where a researcher concentrated on the process, the research then would be conducted as at application level. For instance, a study for assessing urban energy performance using BIM and GIS, where the assessment process is explain in detail, but very few information was given on how the BIM data was inspired by GIS (Yamamura et al., 2017). However, it is very clear that the data level of BIM and GIS integration is very vital for data integration.

\subsection{Data interoperability}

The data level being vital for BIM and GIS integration is also sub divided into geometry and semantic levels. The essence of geometry level is for the purpose of visualization and the semantic level is for analysis as well as visualization, this is one of the few differences between geometry and semantic level, others include; flow of information, information richness etc. in summary, the aim of GIS and BIM integration is data interoperability between the two systems, which is the same as free flow of geometry and semantic information between GIS and BIM.

In this study, concentration is on the achievement of geometry transformation at the geometry level and also, to minimise or to eliminate the mismatch of semantic information at the semantic level. CityGML and IFC are the two most prominent schemas chosen and use as the representative data schemas for GIS and BIM respectively (Deng et al., 2016; Hor et al., 2016). There is need to focus more on the flow of information between the two data formats i.e. sharing and use of information among them.

Data interoperability, which is referred to as the ability to exchange and use information. It is the process that can be considered for the geometry transformation of data from IFC to CityGML or vice versa at the geometry level. In this study we will focus more on FME which is the most widespread and effective commercial platform where ETL is applied (Safe Software, 2018). IFCExplorer, is also a tool that pave way for both unidirectional and bidirectional reading and writing between CityGML and IFC as data schemas (Donkers, 2013). Other custodians' of commercial companies using spatial ETL process for integration of different data from different sources include ORACLE and ESRI, the functions are referred to as ArcGIS data interoperability (ESRI) and Oracle Spatial with
Spatial ETL (Patroumpas, 2014). Consistency should relatively be maintained for geometry and semantic data, during translation between IFC and CityGML by ETL. Never the less, this is based on the understanding of the user for both the two domains (GIS and BIM), particularly for semantic information conversion. But, there is no coordinates information stored in IFC, therefore, there is need for additional step, for instance; LocalCoordinateSystemSetter (inform of georeferencing or Coordinate transformation) in the FME tool in order to set the original location of the model in IFC.

The FME is a data ETL tool that support a wide range of 3D model particularly CityGML. The FME recognise and accept the extraction and loading of data to or from different databases. Patenting to GIS file formats, it accepts Google KML, ESRI shapefiles, also other mapping software that support satellite imaging were equally accepted. It also serves as a barrier that can bridge the gap between different file formats, for instance conversion of LIDAR images to simplified 3D models. Most of the previous research on the extraction and conversion was either conducted manually or semi-automatic but with the advancement in FME these process is to be conducted automatically.

Owing to the limitation on loss of semantic information as a result of BIM and GIS integration, and in addition to the subsequent explanations of data level on BIM and GIS integration. This research proposed a new model that could contribute to increasing the integration of CityGML and IFC. We believe the new model will allow both IFC and CityGML to interact without loss of data and without frequent conversion overheads between IFC and CityGML models. The essence of the approach is to develop a new model that can harness information from both IFC and CityGML. Due to its detailed information model, it enables access to IFC and CityGML as well as integrated access to building information.

The model is defined as a superset model concept that contains the features and objects from both the IFC and CityGML models. All the classes with their concepts will be acquired from both models, also, determine their relationships. Overlapping concepts will be combined and new objects will be created in such a way that both indoor and outdoor objects will be captured. Finally, relationships between the objects will be rebuilt to produce new data model. Similar to IFC and CityGML, Unified Modelling Language (UML) notations will be employed to represent the UML objects and the relationships between them. The model will also develop different LODs. The details in LODs are considered to match those of CityGML. Where, LOD0-LOD4 for CityGML and LOD1-LOD4 for IFC is briefly explained in the previous section. In IFC LOD0 was not shown, because buildings only start to appear at LOD1.

UML accepted and frequently used by many as a modelling language will be employed to serve as the modelling language for the proposed new model. Therefore, the new model will offer free representation for the data model building elements, merging all information from both CityGML and IFC as well populating the missing ones. Hence, they can be all brought into a single data model that can be used for performing all the needed spatial analyses and queries.

\section{CONCLUSION}

GIS and BIM read 3D modelling from two different viewpoints, and are developed using different techniques. Currently, the 
development of GIS and BIM already has some corresponding areas. Thus, the gaps between the two are gradually becoming smaller frequently. Accordingly, at the same time limitations and potentials exist for the future integration of GIS and BIM. GIS can be enriched with its true 3D by being integrated with BIM. The geometry and semantic information transferred from building to geospatial context will positively influence a series of current activities, such as site selection, safety management, and environmental impact assessment.

Though geometry transfer is difficult to actualise, and the semantic level conversion is quite challenging. Few years back, the collective effort on the interpretation of GIS and BIM from a semantic point of view is pretty important, however, the issue of information loss and change is still serious during the information exchange.In order to facilitate the integration of GIS and BIM in relation to data interoperability, this study reviewed papers that were related to this research recently published for the betterment of information exchange within the built environment and other users of GIS and BIM. However, it will be incomplete to conclude without summarizing our few findings of this research, which include the following;

Complementarity of the two systems (GIS and BIM), GIS and BIM complement each other even though, the two system will remain and operate as separate entity also for different purpose.

In addition, the representative of data exchange formats of GIS and BIM are CityGML and IFC respectively, they are the two most popular data exchange formats and the two are simply the agent of data interoperability at the data level

Also, this study highlighted on the geometric transformation between GIS and BIM, precisely from BIM to GIS. However, in order to minimize or eliminate semantic information loss, the geometric transformation between GIS and BIM should be in the opposite direction and later develop a new data model that will encapsulate both CityGML and IFC data models

Even though several researches were conducted on the GIS and BIM integration using different approaches, like, unidirectional to by bidirectional. However, there are still challenges or problems left on solve. These include, transformation between other 3D geometry shapes, like SS, and CSG to b-rep in terms of geometry need to be focus on which is important to achieve for smooth geometry transformation from CityGML. Also, the harmonization of the LODs of CityGML and IFC need more attention to achieve data flow between GIS and BIM. In addition, our future research will concentrate on developing a data model that will be generic as most of the models previously developed are application specific. In essence, the future integration of GIS and BIM is targeting a successful data interoperability between GIS and BIM at the data level in order to have smooth flow of information for a successful visualization and analysis.

\section{REFERENCES}

Amirebrahimi, S. (2015). A data model for integrating GIS and BIM for assessment and 3D visualisation of flood damage to building A Data Model for Integrating GIS and BIM for. Journal of Spatial Science, (August 2016), 10-12. Retrieved from https://www.researchgate.net/publication/281976889

Amirebrahimi, S., Rajabifard, A., Mendis, P., \& Ngo, T. (2016).
A BIM-GIS integration method in support of the assessment and $3 \mathrm{D}$ visualisation of flood damage to a building. Journal of Spatial Science, $\quad 61(2), \quad 317-350$. https://doi.org/10.1080/14498596.2016.1189365

Atazadeh, B., Kalantari, M., Rajabifard, A., Ho, S., \& Ngo, T. (2017). Building Information Modelling for High-rise Land Administration. Transactions in GIS, 21(1), 91-113. https://doi.org/10.1111/tgis.12199

Azhar, S., Khalfan, M., \& Maqsood, T. (2015). Building information modelling (BIM): now and beyond. Construction Economics and Building, 12(4), 15-28. https://doi.org/10.5130/ajceb.v12i4.3032

Aziz, Z., Anumba, C. J., Ruikar, D., Carrillo, P., \& Bouchlaghem, D. (2006). Intelligent wireless web services for construction-A review of the enabling technologies. Automation in Construction, 15(2), 113-123. https://doi.org/10.1016/j.autcon.2005.03.002

Biljecki, F., Ledoux, H., \& Stoter, J. (2016). Generation of Multi-Lod 3D City Models in Citygml With the Procedural Modelling Engine Random3Dcity. ISPRS Annals of Photogrammetry, Remote Sensing and Spatial Information Sciences, IV-4/Wl(September), 51-59. https://doi.org/10.5194/isprs-annals-IV-4-W1-51-2016

Biljecki, F., Stoter, J., Ledoux, H., Zlatanova, S., \& Çöltekin, A. (2015). Applications of 3D City Models: State of the Art Review. ISPRS International Journal of Geo-Information, 4(4), 2842-2889. https://doi.org/10.3390/ijgi4042842

Borrmann, A., Kolbe, T. H., Donaubauer, A., Steuer, H., Jubierre, J. R., \& Flurl, M. (2015). Multi-Scale GeometricSemantic Modeling of Shield Tunnels for GIS and BIM Applications. Computer-Aided Civil and Infrastructure Engineering, 30(4), 263-281. https://doi.org/10.1111/mice. 12090

Costa, G., Sicilia, Á., Lilis, G., Rovas, D., \& Izkara, J. L. (2016). A comprehensive ontologies-based framework to support retrofitting design of energy-efficient districts. Proceedings of the 11th European Conference on Product \& Process Modelling, (September), 673-681. https://doi.org/10.13140/RG.2.2.35829.37603.

Deng, Y., Cheng, J. C. P., \& Anumba, C. (2016). Mapping between BIM and 3D GIS in different levels of detail using schema mediation and instance comparison. Automation in Construction, 67, 1-21. https://doi.org/10.1016/j.autcon.2016.03.006

Donkers, S., Ledoux, H., Zhao, J., \& Stoter, J. (2016). Automatic conversion of IFC datasets to geometrically and semantically correct CityGML LOD3 buildings. Transactions in GIS, 20(4), 547-569. https://doi.org/10.1111/tgis.12162

Dore, C., \& Murphy, M. (2012). Integration of Historic Building Information Modeling ( HBIM ) and 3D GIS for Recording and Managing Cultural Heritage Sites. Proceedings of the 2012 18th International Conference on Virtual Systems and Multimedia, VSMM 2012: Virtual Systems in the Information Society, 369-376. https://doi.org/10.1109/VSMM.2012.6365947 
El-Mekawy, M., Östman, A., \& Hijazi, I. (2012). An Evaluation of IFC-CityGML Unidirectional Conversion. International Journal of Advanced Computer Science and Applications, 3(5), 159-171. https://doi.org/10.14569/IJACSA.2012.030525

Fosu, R., Suprabhas, K., Rathore, Z., \& Cory, C. (2015). Integration of Building Information Modeling ( BIM ) and Geographic Information Systems ( GIS ) - a literature review and future needs. Proc. of the 32nd CIB W78 Conference 2015, 27th-29th October 2015, Eindhoven, The Netherlands, 196204.

Gale, C. G., Singleton, A. D., Bates, A. G., \& Longley, P. A. (2016). Creating the 2011 area classification for output areas (2011 OAC). Journal of Spatial Information Science, 12(12), 1-27. https://doi.org/10.5311/JOSIS.2016.12.232

Gröger, G., \& Plümer, L. (2012). CityGML - Interoperable semantic 3D city models. ISPRS Journal of Photogrammetry and Remote Sensing, 71, 12-33. https://doi.org/10.1016/j.isprsjprs.2012.04.004

Hingmire, V., \& Thomas, N. (2017). GIS \& BIM in Construction - A Timeline Study and the Way Forward, 975982.

Hor, A.-H., Jadidi, A., \& Sohn, G. (2016). Bim-Gis Integrated Geospatial Information Model Using Semantic Web and Rdf Graphs. ISPRS Annals of Photogrammetry, Remote Sensing and Spatial Information Sciences, III-4(July), 73-79. https://doi.org/10.5194/isprsannals-III-4-73-2016

Isikdag, U., Zlatanova, S., \& Underwood, J. (2013). A BIMOriented Model for supporting indoor navigation requirements. Computers, Environment and Urban Systems, 41, 112-123. https://doi.org/10.1016/j.compenvurbsys.2013.05.001

Kang, T. W., \& Hong, C. H. (2015). A study on software architecture for effective BIM/GIS-based facility management data integration. Automation in Construction, 54, 25-38. https://doi.org/10.1016/j.autcon.2015.03.019

Karan, E. P., \& Irizarry, J. (2012). Optimizing Location of Tower Cranes on Construction Sites Through Gis and Bim Integration. Journal of Information Technology in Construction (ITcon), 17(17), 351-366.

Laat, R. De, \& Berlo, L. Van. (2011). Integration of BIM and GIS: The development of the CityGML GeoBIM extension. Advances in 3D Geo-Information Sciences, 211-225. https://doi.org/10.1007/978-3-642-12670-3_13

Liu, X., Wang, X., Wright, G., Cheng, J., Li, X., \& Liu, R. (2017). A State-of-the-Art Review on the Integration of Building Information Modeling (BIM) and Geographic Information System (GIS). ISPRS International Journal of GeoInformation, 6(2), 53. https://doi.org/10.3390/ijgi6020053

Ma, Z., \& Ren, Y. (2017). Integrated Application of BIM and GIS: An Overview. Procedia Engineering, 196(June), 10721079. https://doi.org/10.1016/j.proeng.2017.08.064

Mignard, C., \& Nicolle, C. (2014). Merging BIM and GIS using ontologies application to Urban facility management in ACTIVe3D. Computers in Industry, 65(9), 1276-1290. https://doi.org/10.1016/j.compind.2014.07.008
Niu, S., Pan, W., \& Zhao, Y. (2015). A BIM-GIS Integrated Web-based Visualization System for Low Energy Building Design. Procedia Engineering, 121, 2184-2192. https://doi.org/10.1016/j.proeng.2015.09.091

Patroumpas, K. (2014). TripleGeo: an ETL Tool for Transforming Geospatial Data into RDF Triples. E Workshop Proceedings of the EDBT/ICDT 2014 Joint Conference (March 28, 2014, Athens, Greece) on CEUR- WS.Org (ISSN 16130073), 28-31.

Pauwels, P., Van Deursen, D., Verstraeten, R., De Roo, J., De Meyer, R., Van De Walle, R., \& Van Campenhout, J. (2011). A semantic rule checking environment for building performance checking. Automation in Construction, 20(5), 506-518. https://doi.org/10.1016/j.autcon.2010.11.017

Sjors Donkers. (2013). Automatic generation of CityGML LoD3 building models from IFC models. Delft University of Technology, THe Netherlands.

Song, Y., Wang, X., Tan, Y., Wu, P., Sutrisna, M., Cheng, J., \& Hampson, K. (2017). Trends and Opportunities of BIM-GIS Integration in the Architecture, Engineering and Construction Industry: A Review from a Spatio-Temporal Statistical Perspective. ISPRS International Journal of Geo-Information, 6(12), 397. https://doi.org/10.3390/ijgi6120397

Volk, R., Stengel, J., \& Schultmann, F. (2014). Building Information Modeling (BIM) for existing buildings - Literature review and future needs. Automation in Construction, 38, 109127. https://doi.org/10.1016/j.autcon.2013.10.023

Wu, B., \& Zhang, S. (2016). Integration of GIS And BIM for indoor geovisual analytics. International Archives of the Photogrammetry, Remote Sensing and Spatial Information Sciences - ISPRS Archives, 41(July), 455-458. https://doi.org/10.5194/isprsarchives-XLI-B2-455-2016

Wu, I. C., \& Hsieh, S. H. (2007). Transformation from IFC data model to GML data model: Methodology and tool development. Journal of the Chinese Institute of Engineers, Transactions of the Chinese Institute of Engineers,Series A/Chung-Kuo Kung Ch'eng Hsuch K'an, 30(6), 1085-1090. https://doi.org/10.1080/02533839.2007.9671335

Xie, Y., Wang, M., Liu, X., \& Wu, Y. (2017). Integration of GIS and Moving Objects in Surveillance Video. ISPRS International Journal of Geo-Information, 6(4), 94. https://doi.org/10.3390/ijgi6040094

Yamamura, S., Fan, L., \& Suzuki, Y. (2017). Assessment of Urban Energy Performance through Integration of BIM and GIS for Smart City Planning. Procedia Engineering, 180, 14621472. https://doi.org/10.1016/j.proeng.2017.04.309

Zhu, J., Wright, G., Wang, J., \& Wang, X. (2018). A Critical Review of the Integration of Geographic Information System and Building Information Modelling at the Data Level. ISPRS International Journal of Geo-Information, 7(2), 66. https://doi.org/10.3390/ijgi7020066

\section{Revised August 2018}

